\title{
Guest Editorial MIMO Systems and Applications: Field Experience, Practical Aspects, Limitations and Challenges
}

$\mathbf{O}$ ver the past decade, multiple-input multiple-output (MIMO) technologies have evolved to become an inherent component in all next-generation wireless systems. MIMO technologies will be a part of wireless standards including the cellular systems EV-DO Rev C, UMTS LTE, and the IEEE 802.xx family of standards $802.16 \mathrm{e}, 802.16 \mathrm{j}, 802.16 \mathrm{~m}$, and $802.11 \mathrm{n}$. Future enhancements to these standards, such as those proposed for the IMT Advanced concept, will use MIMO to achieve required data rates in the order of hundreds of Mbps and spectral efficiencies in the order of tens of bps $/ \mathrm{Hz}$.

The field of MIMO research is immense, but it is possible to broadly classify the work in two dimensions. One dimension distinguishes a single MIMO link from a MIMO system with multiple simultaneous links. The former considers a single transmitter with multiple antennas communicating with a single receiver with multiple antennas. The latter considers a many-toone, one-to-many, or many-to-many relationship between transmitters and receivers. The type of wireless networkwhether it be a cellular, mesh, or local area network and whether it be uplink or downlink-determines the multiple access technique, higher layer protocols, and nature of the cochannel interference. While these issues can often be ignored when studying an isolated MIMO link, they must be considered when designing MIMO technologies in a system context.

A second dimension for classifying MIMO research covers the spectrum of development from mathematical theory to prototype. Starting from one end of this spectrum, we have the promise of large information theoretic capacity which can be approached using advanced MIMO transmission and receiver algorithms. These algorithms need to be refined to accommodate real-world impairments like channel estimation errors, frequency offset, and limited bandwidth for feedback. Due to the complexity of MIMO signal processing algorithms, techniques for reducing complexity become essential for implementation in low powered mobile devices. At the other end of the spectrum, the algorithms are implemented in a hardware prototype.

The emphasis of this special issue is on the performance and implementation aspects of MIMO in next-generation wireless networks. With regard to the two dimensions of MIMO research, the 17 papers focus on the system aspects along the first dimension and on the practical implementation aspects along the second dimension. The papers can be grouped into four broad categories:

Digital Object Identifier 10.1109/JSAC.2008.080801

\section{Field Trials and Prototypes}

The papers in this section report on field experience and prototype implementations in the lab.

Implementation and Performance Aspects

The papers in this category address system-level simulations and/or practical MIMO techniques that address issues such as synchronization, power optimization, co-operative diversity, space-time block codes, beam-forming and adaptive transmission.

\section{Techniques to Reduce Complexity}

The papers in this category address reductions in feedback to reduce complexity, the trade-off between complexity reduction and performance and simplified decoding techniques.

Techniques to Address Imperfect Channel Estimates

There are two papers in this category. They discuss the impact of uncertain channel state information (CSI) on transceiver design and the impact of imperfect CSI on power optimization.

\section{Field TRIALS AND PRototypes}

The paper by Taoka, Dai, Higuchi, and Sawahashi presents indoor and field experimental results on achieving $4.92 \mathrm{Gbps}$ in a $100-\mathrm{MHz}$ channel bandwidth in high SNR environments. The system uses OFDM, 12-by-12 MIMO spatial multiplexing, highly efficient modulation and coding, and a novel detection scheme based on maximum likelihood detection.

The paper by Boher, Rabineau and Hélard address real-time implementation aspects related to a 4-by-4 MIMO system. They consider an MMSE-based iterative receiver for MIMOOFDM system and seek to limit the latency and complexity inherent in the iterative process. MMSE equalization implementation is realized using CORDIC operators; the scheduling between MIMO detection and channel decoding is optimized and specific interleaving functions are introduced to reduce latency and accelerate the convergence process. The implemented receiver is integrated in a real-time FPGA test bench and compared in terms of complexity and performance with a non iterative solution.

This paper by Suzuki, Tran, Collings, Daniels, and Hedley analyzes the of performance of a MIMO-OFDM IEEE 802.11n hardware implementation at $5.2 \mathrm{GHz}$ using four transmitters and four receivers. A zero-forcing (ZF) and a list sphere detector (LSD) are compared. They show that the standard assumption of uncorrelated receiver noise does not to agree with measured performance. They explain this discrepancy with the inclusion of transmitter noise. 
The paper by Haene, Perels, and Burg presents a real-time FPGA prototype for a 4-stream MIMO-OFDM transceiver capable of transmitting $216 \mathrm{Mbit} / \mathrm{s}$ in a $20 \mathrm{MHz}$ bandwidth.

\section{ImPlementation AND PERFoRMANCE ASPECTS}

The paper by Wei, Pokhariyal, Sørensen, Kolding, and Mogensen considers Frequency Domain Packet Scheduling (FDPS) problems in a multi-user MIMO system using Spatial Division Multiplexing (SDM). The system-level performance of SDM-FDPS system is evaluated under practical constraints using detailed simulations based on the UTRAN Long Term Evolution (LTE) downlink cellular system framework and the results are compared to derived bounds. The performance results are derived using link level simulations and their subsequent mapping to network level simulations. The paper provides information on various practical aspects that need to be accounted for when evaluating system performance in a realistic MIMO system.

This paper by Zarikoff and Cavers considers a carrier frequency estimation problem when the signal is received from multiple coordinated base stations, where each base station may have a different carrier frequency offset (CFO). The proposed approach uses Newton's method that delivers the CFO vector estimate that maximizes the log-likelihood of the received signal.

Liu, Hou, Shi, and Sherali consider cross-layer optimization problems related to MIMO-based Wireless Mesh Networks (WMN). In particular, the authors consider the problem of jointly optimizing power and bandwidth at each node and multi-hop/multi-path routing in a MIMO-based WMN. They develop a mathematical solution procedure, which combines Lagrangian dual decomposition, gradient projection, cutting plane methods, and sub-gradient methods. They demonstrate that the decoupled structure makes the proposed approach attractive method for MIMO-based mesh networks.

The paper by Papadopoulos and Sundberg develops SpaceTime Block Codes (STBC) for cooperative narrowband and wideband transmissions from multiple non-collocated base stations. In the considered system, the STBC transmit antennas are dispersed over multiple base stations, and the signals from different base stations arrive asynchronously at the receiver. Under these assumptions, they propose techniques that can be used to transform existing STBCs, designed originally for flat fading and synchronous channels, into codes suitable for asynchronous reception.

The paper by Abou-Rjeily and Fawaz considers the problem of Space-Time (ST) coding with Pulse Position Modulation (PPM). While all the existing ST block codes necessitate rotating the phase or amplifying the amplitude of the transmitted symbols, the proposed scheme can be associated with unipolar PPM constellations without introducing any additional constellation extension. The absence of phase rotations renders the proposed scheme convenient for low-cost carrier-less MIMO Time-Hopping Ultra-Wideband (THUWB) systems and for MIMO Free-Space Optical (FSO) communications with direct detection.

The paper by Shen, and Fitz, considers a MIMO-OFDM beamforming design problem from a system level standpoint.
A beamforming method, called the smoothed singular value decomposition (SSVD) algorithm, is proposed which helps improve the receiver channel estimation performance without degrading the benefits of a conventional beamformer. Based on the SSVD algorithm, the Frequency Smoothed Beamformer (FSB) design is then derived, in which smooth effective channels across all sub-carriers are generated and thus the receiver can apply interpolation and smoothing to improve the channel estimation performance. Simulation results show that the FSB design is efficient in the IEEE 802.11n setting.

The paper by Choi and Alamouti considers the issue of adaptive switching between diversity based approaches and spatial multiplexing. It is well known that the full potential of MIMO can only be achieved with adaptive transmission and hence, the study of such switching techniques is very important. In this paper the authors develop a "PHY abstraction" method for switching. In contrast to many ad-hoc approaches, the approach is based on the estimation of packet error events. Hence, the transmission mode is selected to approximately achieve a given error performance. This approach is developed very thoroughly and complexity reductions are also investigated. Results shown demonstrate improved performance relative to other commonly used switching criteria.

The paper by Kalis, Kanatas and Papadias introduces a new perspective to the implementation of wireless MIMO transmission systems with increased bandwidth efficiency. The ideas given here are useful for the implementation of MIMO in customer devices. Unlike traditional spatial multiplexing techniques in MIMO systems, where additional information can be sent through the wireless channel by feeding uncorrelated antenna elements with diverse bit streams, this paper proposes a mapping of diverse bit streams onto orthogonal bases defined in the beam space domain of the transmitting array far-field region. Using this approach it is shown that the capacity of wireless communication systems increases using compact parasitic antenna architectures and a single RF front end at the transmitter, thus paving the way for integrating MIMO systems in cost and size sensitive wireless devices such as mobile terminals and mobile personal digital assistants.

\section{TeChNiques to Reduce CompleXity}

The paper by Khojastepour, Prasad, Wang, Wang, and Madihian considers the issue of reduced complexity feedback and beamforming. With adaptive transmission being of central importance, efficient ways of performing feedback and precoding become critical. This paper employs quantized linear precoding with a novel codebook design that performs well, especially in correlated channels. The design approach aims to maximize the sum-rate while simplifying the feedback computations and requirements. Link and system level simulations are presented for a MIMO-OFDM system and higher throughput with lower complexity is achieved relative to other existing schemes.

The paper by Yoon and Sok-kyu Lee proposes a novel MIMO detection scheme which trades off complexity with performance. Approaching maximum likelihood performance with limited complexity is the fundamental target of MIMO 
receivers and this paper develops an approach based on "polydiagonalization." The idea is to reduce noise inflation in a linear detector by allowing more interference which is then removed by trellis decoding. As the order of the diagonalization approach is increased, performance increases at the expense of complexity. However, the authors demonstrate considerable improvement over traditional approaches even with the simplest implementation of their technique.

The paper by Kim, Lee, and Villasenor, explores performance and complexity tradeoffs in iterative MIMO detection using a sphere decoder and a low-density parity-check (LDPC) decoder. Iterations are performed both within the LDPC decoder as well as via an outer iteration loop through which refined soft information is fed back from the LDPC decoder to a MIMO detector.

\section{TeChNiques to AdDREss IMPERfECt ChAnNel Estimates}

The paper by Shenouda and Davidson, evaluates the design of linear transceivers in the presence of uncertain channel state information.

The paper by Zorba and Pérez-Neira studies scheduling in a broadcast (downlink) channel with partial Channel State Information at the Transmitter (CSIT). Multiple random orthogonal beams are assigned to users in an opportunistic manner. The paper presents a power allocation over the transmitting beams, where a minimum rate per user restriction is required for each scheduled user, standing as a potential Quality of Service (QoS) indicator for the system behavior. Based on the allowed system outage in the QoS achievement, different robust power allocation schemes are proposed, which are efficiently solved through convex optimization tools.

\section{CONCLUding REMARKS}

Despite a large body of research and evidence of successful field trials, much remains to be explored before the use of MIMO reaches its full potential. The following is a non exhaustive list of key areas where MIMO research is required:

Wide area cellular networks suffer from other cell interference. In the presence of this other cell interference, MIMO capacity is very significantly degraded. Therefore, a key question to answer is, "given other cell interference, what is the best way to use a given number of antennas, is it spatial multiplexing, antenna beam-forming, conventional diversity or some adaptive combination of all of these?"

Other cell interference may be mitigated by pre-coding techniques at the transmitter (DPC encoding), or interference cancelation techniques at the receiver or both. However these techniques may also require significant backhaul and also pose a complexity increase. Hence a question arises concerning the extent of the gains that are realizable under practical conditions.

Form factors of devices limit the number of antennas that can be deployed, their layout and spacing. The later may introduce mutual coupling between the antenna elements and thereby reduce the degrees of freedom. Therefore antenna architectures and layout is a key issue to be explored. A related issue for devices is MIMO receiver complexity and its impact on battery life.

The performance of MIMO transmitters and receivers is closely dependent on obtaining channel state information (CSI) and keeping it updated. This is quite a challenge in a practical environment and there may be considerable overhead needed to keep the updated. The fine tuning of MIMO receivers and transmitters via adaptive modulation and coding and the efficient use of CSI is therefore a key area of further research.

The use of MIMO in a relay setting opens up new resource allocation and signal processing challenges, as well as significant future potential. Multiple non-regenerative relay nodes, placed between a MIMO source and a MIMO destination node, can improve the end-to-end MIMO channel properties.

\section{ACKNOWLEDGMENT}

The editors gratefully acknowledge the support of their respective employing organizations to support and facilitate this work. The efforts of many anonymous reviewers are noteworthy; without this work we would not have been able to put this special issue together. We owe special thanks to Ms. Marlene Sealey-Frey, Ms. Laurel Greenidge and Ms. Sue Lange for assistance with this special issue.

\author{
Mansoor Shafi, Guest Editor \\ Telecom New Zealand \\ Wellington, New Zealand \\ Mansoor.shafi@telecom.co.nz
}

Howard Huang, Guest Editor

Bell Labs, Alcatel-Lucent

New Jersey , USA

hchuang@alcatel-lucent.com

Ari Hottinen, Guest Editor
Nokia Research Center
Helsinki, Finland
Ari.hottinen@nokia.com

Peter J. Smith, Guest Editor

Department of Electrical and Computer Engineering

University of Canterbury

Christchurch, New Zealand

peter@elec.canterbury.ac.nz

Reinaldo A. Valenzuela, Guest Editor

Bell Labs, Alcatel-Lucent

New Jersey, USA

rav@alcatel-lucent.com

Leonard J. Cimini, JSAC Board Representative 


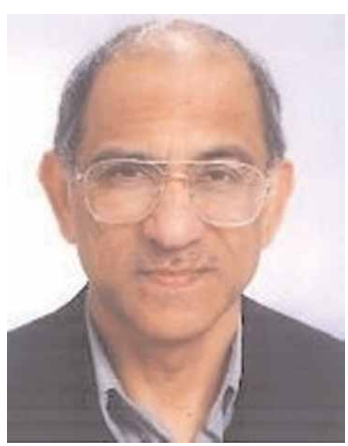

Mansoor Shafi received the B.Sc (Engineering) in Electrical Engineering from Engineering University, Lahore, Pakistan and the $\mathrm{PhD}$ degree from the University of Auckland, Auckland New Zealand in 1970 and 1979 respectively. From 1975-1979 he was a Junior Lecturer at the University of Auckland. Since 1979 he has been with the Telecom New Zealand, where he now holds the position of Principal Advisor Wireless Systems. His research interests are in Wireless Communications. He has published widely in IEEE Journals and IEEE Conferences in the areas of Radio Propagation, Signal processing, MIMO Systems, Adaptive Equalization etc. He was a guest editor of the IEEE JSAC special issue of MIMO systems published in April 2003. His, coauthored, paper, "From Theory to Practice: An Overview of MIMO Space-Time Coded Wireless Systems", published in JSAC April 2003 has won the IEEE Communications Society best tutorial paper award in 2004. Mansoor is a Fellow of the IEEE and is an Adjunct Professor at Canterbury University. He was a Co Chair of the ICC 2005 Wireless Communications Symposium, held in Seoul. In Telecom his role is to advise Telecom Management on the future directions of Wireless Technologies and standards. Dr Shafi is also an editor of the IEEE Transactions Wireless.

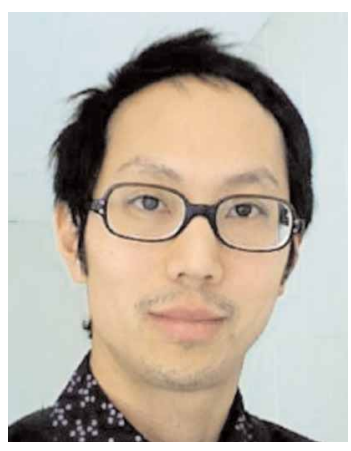

Howard Huang was born in Houston, Texas on March 17, 1969. He received a BSEE degree from Rice University in 1991 and a $\mathrm{Ph} . \mathrm{D}$. in electrical engineering from Princeton University in 1995. Since then, he has been a researcher at Bell Labs, in Holmdel, New Jersey, currently as a Distinguished Member of Technical Staff in the wireless access domain of AlcatelLucent. He has been active in $3 \mathrm{GPP}$ UMTS standards, representing Lucent Technologies when MIMO was first proposed in 2000 and later serving as the rapporteur for the MIMO work item. His research interests include wireless communication theory and system design. He has taught as an adjunct professor at Columbia University and is a Senior Member of the IEEE.

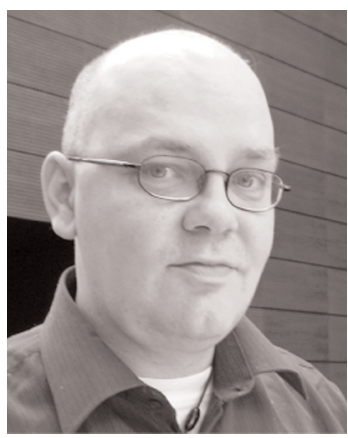

Ari Hottinen graduated with MSc in Applied Mathematics from the University of Helsinki in 1992, and obtained a DSc degree (with distinction) in Computer Science and Engineering in 2004 from Helsinki University of Technology. $\mathrm{He}$ joined Nokia Cellular Systems in 1992 and Nokia Research Center in 1994. Currently, he is a Principal
Member of Research Staff at Nokia Research Center in Helsinki, Finland working on wireless system design, spacetime coding, transmit diversity, detection and estimation, relay networks and cognitive radio. Currently, he is the Nokia representative in an EU STREP project called MASCOT related to multiuser MIMO and relay network research. Dr. Hottinen holds over 50 patents in the area of wireless communications, and he has published over 70 conference and journal papers in wireless communications. He has coauthored the book "Multiantenna transceiver techniques for $3 \mathrm{G}$ and beyond" (Wiley 2003).

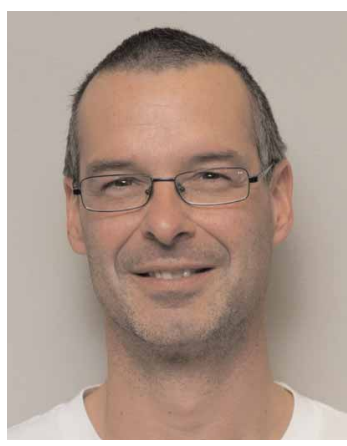

Peter J. Smith received the B.Sc degree in Mathematics and the Ph.D degree in Statistics from the University of London, London, U.K., in 1983 and 1988, respectively. From 1983 to 1986 he was with the Telecommunications Laboratories at GEC Hirst Research Centre. From 1988 to 2001, he was a lecturer in statistics and consulting statistician at Victoria University of Wellington, Wellington, New Zealand. He is currently an associate professor in the Department of Electrical and Computer Engineering at the University of Canterbury, Christchurch, New Zealand. His research interests include the statistical aspects of design and analysis for communication systems, especially antenna arrays, MIMO systems and cognitive radio.

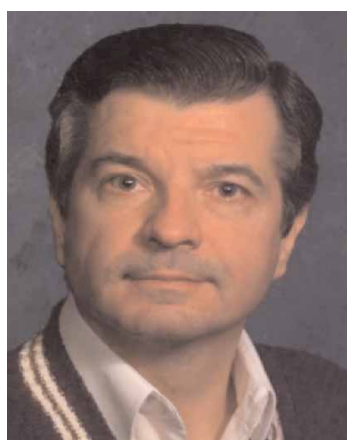

Reinaldo A. Valenzuela obtained his Bachelor of Science from University of Chile and his Ph.D. from the Imperial College of Science and Technology of the University of London, England. At Bell Laboratories, he studied indoor microwave propagation and modeling, packet reservation multiple access for wireless systems and optical WDM networks. He became Manager, Voice Research Dept., at Motorola Codex, involved in the implementation integrated voice and data packet systems. On returning to Bell Laboratories he led a multidisciplinary team to create a software tool for Wireless System Engineering (WiSE), now in widespread use in Lucent Technologies. He received the Distinguished Member of Technical Staff award and is Director of the Wireless Communications Research Department. He is interested in microwave propagation measurements and models, intelligent antennas, third generation wireless system and space time systems achieving high capacities using transmit and receive antenna arrays. He has published over eighty papers and has twelve patents. $\mathrm{He}$ is a Fellow of the IEEE. He is editor for the IEEE Transactions on Communications and the IEEE Transactions on Wireless. 